\title{
ALTERNATIVAS EXPERIMENTAIS NA AVALIAÇÃO DE FAMÍLIAS EM PROGRAMAS DE MELHORAMENTO GENÉTICO DO FEIJOEIRO1
}

\author{
ELAINE APARECIDA DE SOUZA ${ }^{2}$, ISAIAS OLÍVIO GERALDI ${ }^{3}$ e MAGNO ANTONIO PATTO RAMALHO ${ }^{4}$
}

RESUMO - O objetivo deste trabalho foi avaliar o emprego do delineamento em blocos aumentados e de métodos de análise espacial nas etapas iniciais dos programas de melhoramento do feijoeiro (Phaseolus vulgaris $\mathrm{L}$.). Foram avaliadas 121 famílias $\mathrm{S}_{2}$, provenientes de um programa de seleção recorrente, em três locais: Lavras, Lambari e Patos de Minas. Utilizou-se um látice simples 11 x 11, por local. Adicionalmente, foram incluídas duas testemunhas, Carioca e EMGOPA201-Ouro, cada uma alocada a intervalos regulares de cinco em cinco parcelas em cada bloco. A parcela foi composta por duas linhas de dois metros, com 15 sementes por metro. Os dados de produção de grãos (g/parcela) foram submetidos a uma análise de variância considerando os seguintes delineamentos e métodos: látice, blocos aumentados, blocos ao acaso, método de Papadakis, método das médias móveis e testemunha intercalar. Realizou-se a comparação entre as diferentes estratégias quanto à eficiência no controle do erro experimental e em relação à precisão das estimativas de parâmetros genéticos e fenotípicos obtidas em cada método. Constatou-se que o emprego do delineamento em blocos aumentados é viável para a seleção de famílias nas etapas iniciais dos programas de melhoramento genético, principalmente se for aplicada uma intensidade de seleção moderada; porém, esse delineamento não se mostrou apropriado para a estimação de parâmetros genéticos e fenotípicos, em virtude da baixa precisão das estimativas; o uso de testemunha intercalar não proporcionou melhoria na precisão experimental; os métodos de análise de vizinhança, Papadakis e o das médias móveis, foram eficientes no controle da heterogeneidade dos blocos, sendo esta eficiência similar àquela proporcionada pela análise em látice.

Termos para indexação: métodos de melhoramento, parâmetros genéticos, características agronômicas, análises estatísticas, melhoramento de plantas, Phaseolus vulgaris.

\section{EXPERIMENTAL ALTERNATIVES IN THE EVALUATION OF FAMILIES IN THE GENETIC IMPROVEMENT OF BEAN PLANTS}

\begin{abstract}
The aim of this work was to evaluate the viability of using the augmented block designs and spatial analysis methods for early-stage selection in breeding programs for common bean (Phaseolus vulgaris $\mathrm{L}$.). A total of $121 \mathrm{~S}$, progenies were evaluated in three locations: Lavras, Lambari and Patos de Minas, Brazil. A simple 11 x 11 lattice design was employed by location. Additionally, two controls, Carioca and EMGOPA 201-Ouro, were included. The split plots were composed by two $2 \mathrm{~m}$ lines with 15 seeds $/ \mathrm{m}$. The data of the production (g/plot) were submitted to a variance analysis, considering the following strategies: lattice design, augmented block design, randomized complete block design, Papadakis' method, moving means method, and check plots method. The comparison between the different strategies was done considering their efficiency in controlling the experimental error and in relation to the precision of the estimates of genetic and phenotypic parameters obtained in each method. General results indicate that augmented block design is useful in earlier stage of selection, when the intensities of selection are moderate to low; however, this design is not useful for estimation of genetic and phenotypic parameters due to lower precision of estimates; the use of check plots method did not improve the experimental precision; the nearest neighbour, Papadakis and moving means methods were efficient in removing the heterogeneity within blocks; this efficiency was equivalent to lattice analysis for estimation of genetic and phenotypic parameters.
\end{abstract}

Index terms: breeding methods, genetic parameters, agronomic characters, statistical analysis, plant breeding, Phaseolus vulgaris.

\footnotetext{
${ }^{1}$ Aceito para publicação em 26 de julho de 1999.

${ }^{2}$ Eng. Agrôn., Dr., Prof. Adjunto, Dep. de Biologia, Universidade Federal do Lavras (UFLA), Caixa Postal 37, CEP 37200-000 Lavras, MG. E-mail: easouza@ufla.br

${ }^{3}$ Eng. Agrôn., Dr., Prof. Associado, Dep. de Genética, Escola
}

Superior de Agricultura Luiz de Queiroz (ESALQ), Caixa Postal 9, CEP 13418-900 Piracicaba, SP.

E-mail: iogeraldi@carpa.ciagri.usp.br

${ }^{4}$ Eng. Agrôn., Dr., Prof. Titular, Dep. de Biologia, UFLA.

E-mail: magnoapr@ufla.br 


\section{INTRODUÇÃO}

O sucesso de um programa de melhoramento está intimamente ligado à avaliação de um grande número de famílias ou indivíduos, principalmente nas primeiras gerações segregantes. Contudo, no caso do feijoeiro, a avaliação das famílias nas gerações iniciais em experimentos com repetições torna-se difícil, tendo em vista a pequena disponibilidade de sementes nesta fase.

Muitos melhoristas, por essa razão, avaliam as famílias sem conduzir experimentos com repetições. A seleção neste caso é visual. No que se refere ao caráter produção de grãos, que via de regra apresenta herdabilidade reduzida, a eficiência deste método é baixa (Patiño \& Singh, 1989; Silva et al., 1994).

Assim, os melhoristas têm buscado alternativas que contornem tais situações, como o uso de delineamentos em blocos aumentados (Federer, 1956, 1961a, 1961b), o qual permite a avaliação de um grande número de materiais, exigindo menor trabalho e área experimental. A avaliação comparativa desse delineamento tem sido pouco explorada na literatura. Um dos trabalhos com esse enfoque foi realizado com a cultura da batata (Bearzoti, 1994). No caso específico do feijoeiro, há escassez de informação a esse respeito.

Em décadas passadas foram propostas várias metodologias de análises espaciais de experimentos que tornaram-se mais acessíveis aos melhoristas com as facilidades computacionais atuais. Entre esses métodos, o de Papadakis (1937) e o de médias móveis (Townley-Smith \& Hurd, 1973). Recentemente, em razão do interesse nesse tipo de análise, novas propostas surgiram (Bartlett, 1978; Wilkinson et al., 1983; Besag \& Kempton, 1986; Samra et al., 1990; Vivaldi, 1990; Zimmerman \& Harville, 1991; Ball et al., 1993; Brownie et al., 1993; Stroup et al., 1994; Helms et al., 1995; Anoshenko, 1996; Clarke \& Baker, 1996). Em todos esses trabalhos foram evidenciadas as vantagens do uso da análise espacial. Ainda assim, o emprego dessa metodologia de análise nos programas de melhoramento conduzidos no Brasil é escasso.

Foi realizado este trabalho visando comparar a eficiência dessas metodologias na avaliação de famílias em programas de melhoramento do feijoeiro.

\section{MATERIAL E MÉTODOS}

O material utilizado para este estudo constou de 121 famílias $\mathrm{S}_{2}$ de um programa de seleção recorrente com o feijoeiro comum. Estas famílias foram avaliadas em experimentos conduzidos em Lavras, Lambari e Patos de Minas, no Estado de Minas Gerais, com o delineamento látice simples 11 x 11. Adicionalmente, foram incluídas duas testemunhas, as cultivares Carioca e EMGOPA 201-Ouro, alocadas a intervalos regulares de cinco parcelas. Desta forma, foi possível simular em cada repetição do látice um experimento distinto em blocos aumentados, utilizando como tratamentos comuns as duas testemunhas. A parcela foi composta por duas linhas de dois metros, colocando-se 15 sementes por metro.

Foi considerado o caráter produção de grãos (g/parcela), o qual foi submetido a vários métodos de análise de variância, visando avaliar a eficiência de cada método. Inicialmente, as análises foram efetuadas considerando os delineamentos látice e blocos casualizados.

Posteriormente, utilizando a informação de cada repetição, foram efetuadas duas análises de variância por local, no delineamento em blocos aumentados. Para efetuar as comparações entre tratamentos regulares de blocos diferentes, foi estimado um erro efetivo pela expressão desenvolvida por Ferreira (citado por Barbosa, 1996), ou seja:

$\mathrm{QME}_{\mathrm{e}}=\left[1+\frac{1}{r+t-1}+\frac{r}{t(r+t-1)}+\frac{r \sum_{k=1}^{b} n_{k}^{2}}{\operatorname{tn}^{2}(r+t-1)}-\frac{2 \sum_{k=1}^{b} n_{k}^{2}}{\operatorname{tn}(r+t-1)}+\frac{b \sum_{k=1}^{b} n_{k}^{2}}{n^{2}(n+t-1)}\right]=\mathrm{QME}$ em que:

$\mathrm{QME}_{\mathrm{e}}$ : quadrado médio do erro efetivo; r: número de tratamentos regulares;

t: número de tratamentos comuns;

b: número de blocos;

$\mathrm{n}_{\mathrm{k}}$ : número de tratamentos(regulares + comuns) no bloco $\mathrm{k}$; n: número total de parcelas, $\mathrm{n}=\sum_{\mathrm{k}=1}^{\mathrm{b}} \mathrm{n}_{\mathrm{k}}$;

QME: quadrado médio do erro da análise intrablocos.

Outra metodologia avaliada foi a correção por meio das testemunhas intercalares. Para isso, foi obtido um índice ambiental, a partir do desempenho médio das duas testemunhas situadas nas extremidades de cada grupo de cinco parcelas. Utilizando este índice, duas estratégias foram avaliadas. Na primeira, foi efetuada a análise de variância em blocos casualizados, com os dados da parcela ajustados em razão dos desvios do índice ambiental. No segundo caso, foi efetuada uma análise de covariância, também em blocos ao acaso, sendo a covariável o índice ambiental.

Além disso, os dados foram analisados pelo método de Papadakis (1937), e pelo método das médias móveis 
(Towley-Smith \& Hurd, 1973), sendo utilizado como ajuste o desempenho médio de seis parcelas vizinhas, três à direita e três à esquerda da parcela de referência.

A comparação desses procedimentos foi realizada considerando as estimativas de parâmetros genéticos e fenotípicos, tais como coeficiente de variação experimental, herdabilidade no sentido amplo ao nível de média de família e seus respectivos intervalos de confiança, estimados pela expressão apresentada por Knapp et al. (1985). Além disso, foram utilizados a correlação classificatória de Spearman e dois outros critérios. O primeiro deles, proposto por Hamblin \& Zimmermann (1986), diz respeito à estimativa da eficiência de seleção de cada experimento, ou seja, à proporção de famílias que cada tipo de metodologia estatística de avaliação das famílias consegue identificar, e que são coincidentes com as famílias selecionadas no tipo tomado como padrão, que para este caso foi o látice. A expressão que permite estimar essa eficiência de seleção (ES) é:

$\mathrm{ES}(\%)=100(\mathrm{~A}-\mathrm{C}) /(\mathrm{B}-\mathrm{C})$

em que:

A: número de famílias selecionadas tanto na metodologia estatística considerada, como na metodologia-padrão;

B: número de famílias selecionadas;

C: número de famílias esperado por simples coincidência, unicamente pelo acaso, que é igual a $10 \%$ de B.

A outra metodologia é o índice de diferenciação de Fasoulas (1983), o qual é estimado pela expressão $\mathrm{D}=200 \sum \mathrm{f} / \mathrm{n}(\mathrm{n}-1)$, sendo $\mathrm{n}$ o número total de famílias e f o número de médias que uma determinada família supera estatisticamente, após a aplicação de um teste de médias. Neste trabalho, utilizou-se o teste Duncan, para verificação das diferenças entre médias. Este índice (D) fornece o porcentual de diferenças estatísticas entre médias que o método estatístico considerado consegue detectar.

\section{RESULTADOSE DISCUSSÃO}

As estimativas do coeficiente de variação $(\mathrm{CV})$ obtidas nos três locais e nas análises conjuntas são apresentadas nas Tabelas 1 e 2. Observa-se que as estimativas do $\mathrm{CV}$ foram elevadas em relação a todas as estratégias de análise de variância. Nas análises de variância por local elas apresentaram variação de $20,94 \%$ no delineamento em blocos aumentados (BA1), e de $45,02 \%$, no delineamento em blocos ao acaso ajustado pela testemunha intercalar, ambas em Lambari. O CV desses experimentos foi de magnitude superior ao que normalmente é encontrado em
TABELA 1. Coeficientes de variação (CV) da produção de grãos (g/parcela) do feijoeiro em todos os métodos estatísticos avaliados nos experimentos em Lavras, Lambari e Patos.

\begin{tabular}{lccc}
\hline Delineamento $^{1}$ & \multicolumn{3}{c}{ CV $(\%)$} \\
\cline { 2 - 4 } & Lavras & Lambari & Patos \\
\hline Látice & 35,83 & 36,33 & 25,75 \\
BA1 & 38,78 & 20,94 & 27,07 \\
BA2 & 28,41 & 31,85 & 35,49 \\
DBC & 36,88 & 38,98 & 26,47 \\
Test1 & 43,25 & 45,02 & 42,94 \\
Test2 & 36,91 & 39,14 & 26,45 \\
Papadakis & 31,81 & 30,59 & 25,92 \\
Médias móveis & 32,03 & 29,69 & 25,08 \\
\hline
\end{tabular}

1 Análises de variância: Látice - em látice; BA1 e BA2 - em blocos aumentados utilizando os dados das repetições 1 e 2, respectivamente; DBC em blocos ao acaso; Test1 - em blocos ao acaso ajustado pela testemunha intercalar. Análises de covariância em blocos ao acaso: Test2 - utilizando como covariável a testemunha intercalar; Papadakis - utilizando o método de Papadakis (1937); Médias móveis - utilizando como covariável a média móvel.

experimentos com a cultura do feijoeiro na região (Abreu et al., 1994). Deve ser salientado que nesses trabalhos a parcela era de dimensões bem superiores à utilizada. Tratando-se de famílias em gerações iniciais, a disponibilidade de sementes e de área experimental quase sempre restringem o tamanho das parcelas. Além disso, na avaliação de famílias, o QMErro apresenta, além da variância ambiental entre parcelas, a variância fenotípica dentro de parcelas. Logo, é de se esperar que as estimativas do QMErro nesses casos sejam superiores, principalmente nas gerações iniciais. Tal fato tem sido constatado em outros experimentos utilizando famílias nas primeiras gerações (Souza, 1993; Collicchio, 1995; Gonçalves, 1995).

As estratégias de análise de variância que utilizaram o ajustamento com base na testemunha intercalar mostraram-se incapazes de remover os efeitos ambientais visando melhorar a precisão experimental, sendo que o método de ajustamento antes da realização da análise de variância em blocos ao acaso (Test1) foi menos eficiente que o método da análise de covariância (Test 2). Os métodos de análise de vizinhança proporcionaram uma redução razoável no $\mathrm{CV}$, cerca de $18 \%$ em relação ao blocos ao acaso e $13 \%$ em relação ao látice (Tabela 2 ). Já o delineamento em blocos aumentados apresen- 
TABELA 2. Resumo da análise de variância conjunta e estimativas de herdabilidade e seus respectivos intervalos de confiança, da produção de grãos (g/parcela) do feijoeiro em todos os métodos estatísticos avaliados.

\begin{tabular}{|c|c|c|c|c|c|c|c|}
\hline Delineamento $^{1}$ & $\begin{array}{c}\mathrm{F} \\
\text { (Famílias) } \\
\end{array}$ & $\begin{array}{c}\mathrm{F} \\
\text { (Famílias x locais) } \\
\end{array}$ & $\mathrm{CV}(\%)$ & Média & $\mathrm{h}^{2}(\%)$ & $\begin{array}{l}\text { Limite } \\
\text { inferior }\end{array}$ & $\begin{array}{c}\text { Limite } \\
\text { superior }\end{array}$ \\
\hline Látice & $1,82 * *$ & $1,15^{\mathrm{ns}}$ & 33,93 & 192,2 & 45,08 & 25,64 & 60,09 \\
\hline B A & $1,23^{\mathrm{ns}}$ & $1,76^{*}$ & 34,19 & 190,0 & 18,98 & $-9,69$ & 41,12 \\
\hline $\mathrm{DBC}$ & $1,81 * *$ & $1,10^{\mathrm{ns}}$ & 35,40 & 192,2 & 44,82 & 25,31 & 59,91 \\
\hline Test 1 & $1,72 * *$ & $0,95^{\mathrm{ns}}$ & 44,02 & 192,4 & 42,09 & 21,60 & 57,92 \\
\hline Test2 & $1,80 * *$ & $1,11^{\mathrm{ns}}$ & 35,46 & 192,2 & 44,63 & 25,03 & 59,76 \\
\hline Papadakis & $1,73 * *$ & $1,37 *$ & 30,28 & 192,2 & 42,26 & 21,83 & 58,04 \\
\hline Médias móveis & $1,78 * *$ & $1,34 *$ & 29,94 & 192,2 & 43,70 & 23,78 & 59,09 \\
\hline
\end{tabular}

${ }^{1}$ Análises de variância: Látice - em látice; BA: análise conjunta no delineamento em blocos aumentados utilizando as médias de famílias de BA1 em Lavras, BA1 em Lambari e BA2 em Patos, respectivamente; DBC - em blocos ao acaso; Test1 - em blocos ao acaso ajustado pela testemunha intercalar. Análises de covariância em blocos ao acaso: Test2 - utilizando como covariável a testemunha intercalar; Papadakis - utilizando o método de Papadakis (1937); Médias móveis - utilizando como covariável a média móvel.

ns, * e ** Não-significativo e significativo a $1 \%$ e $5 \%$ de probabilidade, pelo teste de $\mathrm{F}$, respectivamente.

tou um CV semelhante ao látice e ao delineamento em blocos ao acaso.

As estimativas de herdabilidade foram semelhantes entre as estratégias estatísticas em que se utilizaram repetições. Nesses casos, pode ser constatado que os valores dos limites dos intervalos de confiança foram bem próximos (Tabela 2). Esses resultados não são coincidentes com os obtidos nas análises de variância individuais (Tabela 3), o que sugere que apesar de a interação famílias x locais não ter sido significativa na maioria das estratégias de análise de variância, sua participação na estimativa dos componentes de variância foi considerável, confirmando, assim, as observações feitas por Rosielle (1980) e Helms et al. (1995). Esses autores verificaram que, sendo a diferença entre os métodos, evidente, em um local isolado, não o será na análise de variância conjunta, pela interação genótipos x ambientes. Contudo, os resultados obtidos neste trabalho são semelhantes aos valores relatados na literatura (Ramalho et al., 1979a, 1979b; Santos et al., 1985; Singh et al., 1990; Collichio, 1995; Gonçalves, 1995). Quanto ao delineamento em blocos aumentados, observa-se que o valor da estimativa de $\mathrm{h}^{2}$ é bem discrepante em relação aos demais, e que não há concordância entre os valores das estimativas de herdabilidade e seus respectivos intervalos de confiança, em comparação com as obtidas pelos demais métodos.
Os coeficientes de correlação classificatória de Spearman (Tabela 4) obtidos foram elevados, o que mostra que não houve grandes diferenças entre o látice e as demais estratégias, à exceção do delineamento em blocos aumentados.

A eficiência de seleção avaliada pela expressão de Hamblin \& Zimmermann (1986) foi estimada considerando-se diferentes intensidades de seleção, a saber: $16 \%$ (seleção das 20 famílias superiores e 20 inferiores), 33\% (seleção das 40 famílias superiores) e 50\% (seleção das 60 famílias superiores) respectivamente, em relação ao látice. Constata-se, pelos resultados obtidos, que houve uma boa concordância entre as estratégias de análise de variância em que se utilizaram repetições. Porém, no delineamento em blocos aumentados a eficiência de seleção foi menor (Tabela 4). Tais resultados são concordantes com os obtidos pela correlação de Spearman, e provavelmente decorrem do fato de as médias no delineamento em blocos aumentados serem estimadas a partir de uma só repetição, o que afeta o erro-padrão das médias das famílias, e, conseqüentemente, sua classificação. A partir daí, é necessária a condução de experimentos com repetições, pois a seleção das melhores famílias deve ser efetuada com maior rigor.

O índice de diferenciação do delineamento em blocos aumentados foi inferior ao obtido pelas demais estratégias (Tabela 4). Considerando este delineamento, dos 7.260 pares de contrastes possíveis en- 
TABELA3. Estimativas de herdabilidade e seus respectivos intervalos de confiança, da produção de grãos (g/parcela) do feijoeiro, em todos os métodos estatísticos avaliados em Lavras, Lambari e Patos.

\begin{tabular}{|c|c|c|c|c|c|c|c|c|c|}
\hline \multirow[t]{2}{*}{ Delineamento $^{1}$} & \multicolumn{3}{|c|}{ Lavras } & \multicolumn{3}{|c|}{ Lambari } & \multicolumn{3}{|c|}{ Patos } \\
\hline & $\mathrm{h}^{2}(\%)$ & $\begin{array}{l}\text { Limite } \\
\text { inferior }\end{array}$ & $\begin{array}{l}\text { Limite } \\
\text { superior }\end{array}$ & $\mathrm{h}^{2}(\%)$ & $\begin{array}{l}\text { Limite } \\
\text { inferior }\end{array}$ & $\begin{array}{c}\text { Limite } \\
\text { superior }\end{array}$ & $\mathrm{h}^{2}(\%)$ & $\begin{array}{l}\text { Limite } \\
\text { inferior }\end{array}$ & $\begin{array}{l}\text { Limite } \\
\text { superior }\end{array}$ \\
\hline Látice & 35,53 & 5,68 & 55,65 & 11,68 & $-2,92$ & 39,24 & 48,67 & 24,90 & 64,68 \\
\hline BA1 & 27,31 & 27,31 & 58,69 & 70,51 & 40,73 & 83,24 & 48,00 & $-4,52$ & 70,45 \\
\hline BA2 & 60,58 & 20,76 & 77,60 & 45,14 & $-10,26$ & 68,83 & 61,88 & 23,38 & 78,34 \\
\hline DBC & 30,12 & 5,52 & 51,22 & 13,58 & $-16,83$ & 39,68 & 44,70 & 25,25 & 61,41 \\
\hline Test 1 & 30,52 & 6,08 & 51,51 & $-3,82$ & $-40,36$ & 27,53 & $-2,53$ & $-38,61$ & 28,43 \\
\hline Test2 & 30,34 & 0,10 & 51,40 & 11,64 & $-26,69$ & 38,37 & 44,44 & 20,33 & 61,24 \\
\hline Papadakis & 45,30 & 21,55 & 61,84 & 24,16 & $-8,76$ & 47,09 & 41,12 & 15,58 & 58,93 \\
\hline Médias móveis & 40,64 & 14,89 & 58,60 & 29,64 & $-0,90$ & 50,92 & 43,92 & 19,59 & 60,88 \\
\hline
\end{tabular}

${ }^{1}$ Análises de variância: Látice - em látice; BA1 e BA2 - em blocos aumentados utilizando os dados das repetições 1 e 2, respectivamente; DBC - em blocos ao acaso; Test 1 - em blocos ao acaso ajustado pela testemunha intercalar. Análises de covariância em blocos ao acaso: Test2 - utilizando como covariável a testemunha intercalar; Papadakis - utilizando o método de Papadakis (1937); Médias móveis - utilizando como covariável a média móvel.

TABELA 4. Coeficientes de correlação de Spearman, eficiência de seleção (\%) entre as médias estimadas no látice e nos demais métodos de análise de variância e índice de diferenciação de Fasoulas (D\%) da produção de grãos do feijoeiro.

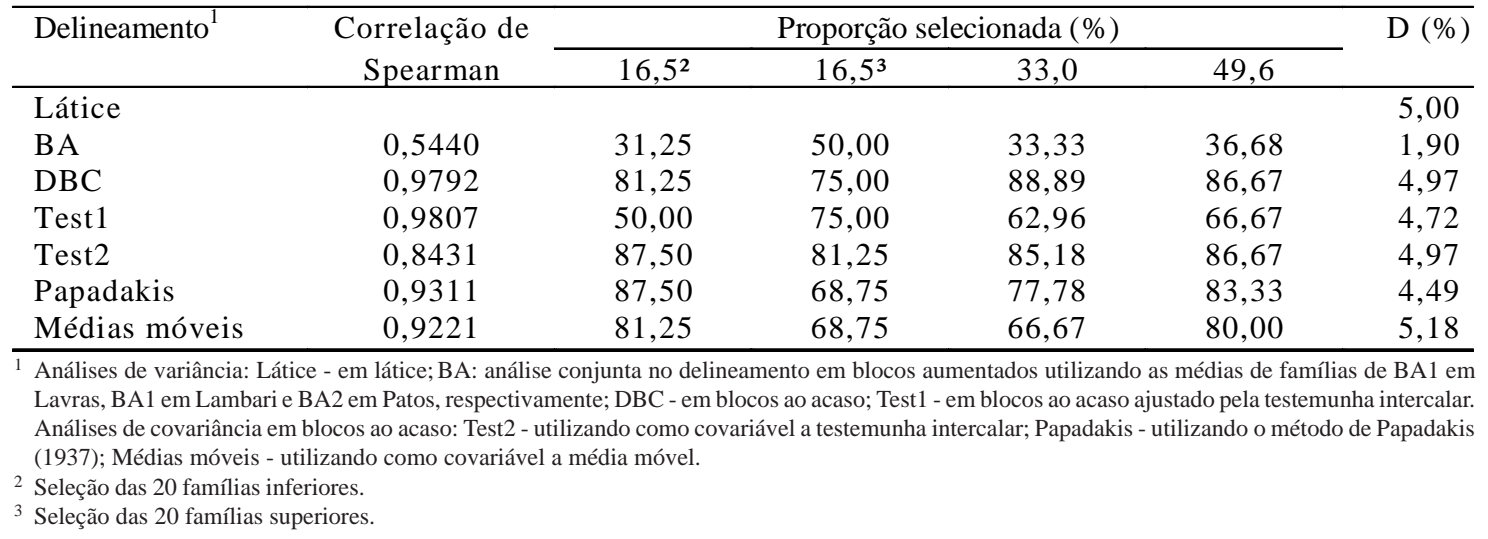

tre as 121 médias, apenas $1,9 \%$ foram significativos, ou seja, 138 contrastes, enquanto o látice conseguiu detectar diferenças significativas entre 363 pares de comparações $(\mathrm{D}=5 \%)$.

Os resultados obtidos indicam que o delineamento em blocos aumentados é uma alternativa viável para a avaliação de um grande número de genótipos nas etapas iniciais dos programas de melhoramento visando principalmente ao descarte de materiais, desde que sejam aplicadas intensidades de seleção brandas.

Para melhorar a eficiência deste delineamento, alguns aspectos têm que ser considerados, como a utilização de testemunhas adequadas, que representem bem a variação ambiental da população segregante em estudo, procurando-se, dentro do possível, utilizar alguns dos genótipos que estejam sendo avaliados, como realçado por Bearzoti (1994).

Por outro lado, a menor precisão das estimativas dos parâmetros genéticos obtidas com o uso desse delineamento não chega a comprometer sua utilidade, nas fases para as quais é recomendado.

Os métodos de análise de vizinhança (Papadakis e médias móveis) mostraram-se promissores na remoção de efeitos ambientais, com base na correlação entre parcelas adjacentes, quando se detectou 
heterogeneidade dentro dos blocos. Nesses casos, a melhoria na precisão experimental foi, na maioria das vezes, semelhante à do látice, mostrando, assim, que o controle local em linhas e/ou colunas foi tão efetivo quanto os métodos baseados na análise espacial.

\section{CONCLUSÕES}

1. O emprego do delineamento em blocos aumentados é viável para a seleção de famílias nas etapas iniciais dos programas de melhoramento genético, principalmente se for aplicada uma intensidade de seleção moderada; porém, esse delineamento não é apropriado para a estimação de parâmetros genéticos e fenotípicos, pela baixa precisão das estimativas.

2. O uso de testemunha intercalar não proporciona melhoria na precisão experimental.

3. Os métodos de análise de vizinhança e o das médias móveis são eficientes no controle da heterogeneidade dos blocos, sendo esta eficiência similar à proporcionada pela análise em látice.

\section{AGRADECIMENTOS}

Ao Programa de Melhoramento Genético do Feijoeiro do Departamento de Biologia/UFLA (Universidade Federal de Lavras), FAPEMIG (Fundação de Amparo à Pesquisa do Estado de Minas Gerais) e CAPES (Coordenadoria de Aperfeiçoamento de Pessoal de Nível Superior).

\section{REFERÊNCIAS}

ABREU, A. de F.B.; RAMALHO, M.A.P.; SANTOS, J.B. dos; MARTINS, L.A. Progresso do melhoramento genético do feijoeiro nas décadas de setenta e oitenta nas regiões Sul e Alto Paranaíba em Minas Gerais. Pesquisa Agropecuária Brasileira, Brasília, v.29, n.1, p.105-112, jan. 1994.

ANOSHENKO, B.Y. Local adjustment method for field experiments. 1 . The method and its examination by computer simulation. Euphytica, Dordrecht, v.90, n.2, p.137-148, 1996
BALL, S.T.; MULLA, D.J.; KONZAK, C.F. Spatial heterogeneity effects variety trial interpretation. Crop Science, Madison, v.33, p.931-935, 1993.

BARBOSA, M.H.P. Capacidade combinatória e comparação entre critérios de seleção de clones de batata (Solanum tuberosum L.). Lavras : UFLA, 1996. 141p. Tese de Doutorado.

BARTLETT, M.S. Nearest neighbour models in the analysis of field experiments with large blocks. Royal Statistical Society Journal, Serie B, Oxford, v.40, n.2, p.147-174, 1978.

BEARZOTI, E. Comparação entre métodos estatísticos na avaliação de clones de batata em um programa de melhoramento. Lavras : ESAL, 1994. 128p. Dissertação de Mestrado.

BESAG, J.; KEMPTON, R. Statistical analysis of field experiments using neigbouring plots. Biometrics, Washington, v.42, p.231-251, 1986.

BROWNIE, C.; BOWMAN, D.T.; BURTON, J.W. Estimating spatial variation in analysis of data from yield trials: a comparison of methods. Agronomy Journal, Madison, v.85, p.1244-1253, 1993.

CLARKE, F.R.; BAKER, R.J. Spatial analysis improves precision of seed lot comparisons. Crop Science, Madison, v.36, p.1180-1184, 1996.

COLLICCHIO, E. Associação entre o porte da planta do feijoeiro e o tamanho dos grãos. Lavras : UFLA, 1995. 98p. Dissertação de Mestrado.

FASOULAS, A.C. Rating cultivars and trials in applied plant breeding. Euphytica, Dordrecht, v.32, n.3, p.939-943, 1983.

FEDERER, W.T. Augmented designs with one-way elimination of heterogeneity. Biometrics, Washington, v.17, n.3, p.447-473, 1961a.

FEDERER, W.T. Augmented designs with two three and higher was elimination of heterogeneity (abstract). Biometrics, Washington, v.17, n.3, p.166, 1961b.

FEDERER, W.T. Augmented (hoonuiaku) designs. Hawaiian Planters' Record, Aiea, v.55, p.191-208, 1956.

GONÇALVES, P.R. Obtenção de linhagens de feijoeiro (Phaseolus vulgaris) com grãos tipo Carioca e resistente à antracnose e mancha angular. Lavras : UFLA, 1995. 65p. Dissertação de Mestrado. 
HAMBLIN, J.; ZIMMERMANN, M.J. de O. Breeding common bean for yield mixtures. Plant Breeding Reviews, New York, v.4, p. 245-272, 1986.

HELMS, T.C.; ORF, J.H.; SCOTT, R.A. Nearest-neighboradjusted means as a selection criterion within two soybean populations. Canadian Journal of Plant Science, Ottawa, v.75, p.857-863, 1995.

KNAPP, S.J.; STROUP, W.W.; ROSS, W.M. Exact confidence intervals for heritability on a progeny mean basis. Crop Science, Madison, v.25, p.192194, 1985.

PAPADAKIS, J.S. Méthod statistique pour des experiences sur champ. Thessalonike : Institut d'Amélioration des Plantes à Salonique, 1937. 30p. (Bullettin, 23).

PATIÑO, H.; SINGH, S.P. Visual selection for seed yield in the $\mathrm{F}_{2}$ and $\mathrm{F}_{3}$ generations of nine common bean crosses. Bean Improvement Cooperative Annual Report, East Lansing, v.32, p.79-80, 1989.

RAMALHO, M.A.P.; ANDRADE, L.A.; TEIXEIRA, N.C.S. Correlações genéticas e fenotípicas entre caracteres do feijão (Phaseolus vulgaris L.). Ciência e Prática, Lavras, v.3, n.1, p.63-70, 1979a.

RAMALHO, M.A.P.; SANTOS, J.B. dos; SANTA CECÍLIA, F.C.; ANDRADE, M.A. Seleção de progênies de feijão "Pintado" e estimativas dos parâmetros genéticos e fenotípicos. Ciência e Prática, Lavras, v.3, n.1, p. 51-57, 1979b.

ROSIELLE, A.A. Comparasion of lattice designs, check plots, and moving means in wheat breeding trials. Euphytica, Dordrecht, v.29, p.129-133, 1980.

SAMRA, J.S.; ANLAUF, R.; WEBER, W.E. Spatial dependence of growth attributes and local control in wheat and oat breeding experiments. Crop Science, Madison, v.30, p.1200-1205, 1990.
SANTOS, J.B. dos; VENCOVSKY, R.; RAMALHO, M.A.P. Controle genético da produção de grãos e seus componentes primários em feijoeiro. Pesquisa Agropecuária Brasileira, Brasília, v.20, n.10, p.1203-1211, out. 1985.

SILVA, H.D.; RAMALHO, M.A.P.; ABREU, A.F.B.; MARTINS, L.A. Efeito da seleção visual para produtividade de grãos em populações segregantes do feijoeiro. II. Seleção entre famílias. Ciência e Prática, Lavras, v.18, n.2, p.181-185, 1994.

SINGH, S.P.; LEPIZ, R.; GUTIERREZ, S.A.; URREA, C.; MOLINA, A.; TERAN, H. Yield testing of early generation population of common bean. Crop Science, Madison, v.30, p.874-878, 1990.

SOUZA, E.A. Controle genético de alguns caracteres do feijoeiro. Lavras : ESAL, 1993. 105p. Dissertação de Mestrado.

STROUP, W.W.; BAENZIGER, P.S.; MULITZE, D.K. Removing spatial variation from wheat yield trials: a comparison of methods. Crop Science, Madison, v.86, p.62-66, 1994.

TOWNLEY-SMITH, T.F.; HURD, W.A. Use of moving means in wheat yields trials. Canadian Journal of Plant Science, Ottawa, v.53, p.447-450, 1973.

VIVALDI, L.J. Comparação entre métodos de análise espacial de experimentos de campo. Pesquisa Agropecuária Brasileira, Brasília, v.25, n.1, p.7784, jan. 1990.

WILKINSON, G.N.; ECHERT, S.R.; HOANCOCK, T.W.; MAYO, O. Nearest neighbour (NN) analysis of field experiments. Royal Statistical Society Journal, Serie B, Oxford, v.40, p.151-211, 1983.

ZIMMERMAN, D.L.; HARVILLE, D.A. A random field approach to the analysis of field-plot experiments and other spatial experiments. Biometrics, Washington, v.47, p.223-239, 1991 